\title{
Genetic Markers Can Predict Chondrogenic Differentiation Potential in Bone Marrow-Derived Mesenchymal Stromal Cells
}

\author{
Masami Kanawa, ${ }^{1}$ Akira Igarashi, ${ }^{2,3}$ Katsumi Fujimoto ${ }^{D},{ }^{3,4}$ Yukihito Higashi, ${ }^{5}$ \\ Hidemi Kurihara, ${ }^{6}$ Masaru Sugiyama, ${ }^{7}$ Tania Saskianti, ${ }^{3,8}$ Yukio Kato ${ }^{1 D},{ }^{3}$ \\ and Takeshi Kawamoto ${ }^{1 D}{ }^{3,9}$
}

\author{
${ }^{1}$ Natural Science Center for Basic Research and Development, Hiroshima University, Hiroshima 734-8533, Japan \\ ${ }^{2}$ Department of Advanced Technology and Development, BML, Inc., Saitama 350-1101, Japan \\ ${ }^{3}$ Department of Dental and Medical Biochemistry, Graduate School of Biomedical \& Health Sciences, Hiroshima University, \\ Hiroshima 734-8533, Japan \\ ${ }^{4}$ Department of Molecular Biology and Biochemistry, Graduate School of Biomedical \& Health Sciences, Hiroshima University, \\ Hiroshima 734-8533, Japan \\ ${ }^{5}$ Research Center for Radiation Genome Medicine, Research Institute for Radiation Biology and Medicine, Hiroshima University, \\ Hiroshima 734-8533, Japan \\ ${ }^{6}$ Departments of Periodontal Medicine, Graduate School of Biomedical \& Health Sciences, Hiroshima University, \\ Hiroshima 734-8533, Japan \\ ${ }^{7}$ Departments of Public Oral Health, Graduate School of Biomedical \& Health Sciences, Hiroshima University, \\ Hiroshima 734-8533, Japan \\ ${ }^{8}$ Department of Pediatric Dentistry, Faculty of Dental Medicine, Universitas Airlangga, Surabaya 60132, Indonesia \\ ${ }^{9}$ Writing Center, Hiroshima University, Higashi-Hiroshima 739-8512, Japan
}

Correspondence should be addressed to Takeshi Kawamoto; tkawamo@hiroshima-u.ac.jp

Received 29 May 2018; Accepted 27 August 2018; Published 10 October 2018

Academic Editor: Stan Gronthos

Copyright ( 2018 Masami Kanawa et al. This is an open access article distributed under the Creative Commons Attribution License, which permits unrestricted use, distribution, and reproduction in any medium, provided the original work is properly cited.

\begin{abstract}
The precise predictions of the differentiation direction and potential of mesenchymal stromal cells (MSCs) are an important key to the success of regenerative medicine. The expression levels of fate-determining genes may provide tools for predicting differentiation potential. The expression levels of 95 candidate marker genes and glycosaminoglycan (GAG) contents after chondrogenic induction in 10 undifferentiated ilium and 5 jaw MSC cultures were determined, and their correlations were analyzed. The expression levels of eight genes before the induction of chondrogenic MSC differentiation were significantly correlated with the GAG levels after induction. Based on correlation patterns, the eight genes were classified into two groups: group 1 genes (AURKB, E2F1, CDKN2D, LIF, and ACLY), related to cell cycle regulation, and group 2 genes (CD74, EFEMP1, and TGM2), involved in chondrogenesis. The expression levels of the group 2 genes were significantly correlated with the ages of the cell donors. The expression levels of CDKN2D, CD74, and TGM2 were >10-fold higher in highly potent MSCs (ilium MSCs) than in MSCs with limited potential (jaw MSCs). Three-dimensional (3D) scatter plot analyses of the expression levels of these genes showed reduced variability between donors and confirmed predictive potential. These data suggest that group 2 genes are involved in age-dependent decreases in the chondrogenic differentiation potential of MSCs, and combined 3D analyses of the expression profiles of three genes, including two group 2 genes, were predictive of MSC differentiation potential.
\end{abstract}

\section{Introduction}

Mesenchymal stromal cells (MSCs) can differentiate into various cell types, including osteoblasts, chondrocytes, or adipocytes; therefore, they are promising as regenerative medicine [1-4]. MSCs are usually obtained using bone marrow aspirated from the iliac crest. Recently, we developed another method to obtain MSCs from jaw bone marrow 
TABLE 1: Candidate prediction marker genes showing significant positive correlation between their expression levels in undifferentiated MSCs and GAG contents after chondrogenic induction.

\begin{tabular}{lcl}
\hline Gene & Full name & Probe set ID \\
\hline ACLY & ATP citrate lyase & Hs00153764_m1 \\
AURKB & Aurora kinase B & Hs00177782_m1 \\
CD74 & CD74 molecule & Hs00269961_m1 \\
CDKN2D & Cyclin-dependent kinase inhibitor 2D & Hs00176481_m1 \\
E2F1 & E2F transcription factor 1 & Hs00153451_m1 \\
EFEMP1 & EGF containing fibulin extracellular matrix protein 1 & Hs00251661_m1 \\
LIF & Leukemia inhibitory factor & Hs00171455_m1 \\
TGM2 & Transglutaminase 2 & Hs00190278_m1 \\
\hline
\end{tabular}

$r$ : Pearson correlation coefficient; ${ }^{*} P<0.05$.

collected during wisdom tooth extraction [5], a surgery that several young adults undergo. The differentiation potential of MSCs varies depending on the tissue sources and the physical conditions of donors [6-9]. Accordingly, we previously demonstrated that MSCs from jaw bone marrow have poor chondrogenic differentiation capacity, although they have high osteogenic differentiation capacity, as seen in ilium MSCs [5]. In another study, we showed that chondrogenic differentiation potential of MSCs from ilium bone marrow depends on the age of cell donors [10].

Predicting the differentiation direction of MSCs is a crucial determinant of clinical outcomes of regenerative medicine, and several cell surface markers have been identified as predictors of such capabilities. $\mathrm{CD}_{105}{ }^{+}, \mathrm{CD}_{146}{ }^{+}$, $\mathrm{CD} 271^{+}$, or ROR2 ${ }^{+}$MSCs have enhanced capacity for chondrogenic differentiation [11-14]. CD146 ${ }^{+}$MSCs also have greater therapeutic potential than $\mathrm{CD}_{146^{-}}$cells [15]. However, the utility of these cell surface markers has not yet been established. Hence, in addition to cell surface markers, gene expression patterns may provide a strategy for predicting the differentiation potential of MSCs.

Recently, we produced a TaqMan low-density array comprising real-time PCR probes and the primers for 95 marker candidate genes that were selected from microarray analyses of 17,703 genes [16]. Because these 95 genes showed higher expression levels in MSCs than in fibroblasts, we predicted that some of these genes may serve as MSC markers for identifying cells having high potential for differentiation into specific cell types such as chondrocytes.

In the present study, we aimed to find prediction markers to select potent MSCs by comparing gene expression profiles and differentiation levels. Accordingly, we determined the expression levels of 95 marker candidate genes in undifferentiated MSCs from various donors and analyzed the correlation between the expression and glycosaminoglycan (GAG) protein levels in MSCs after induction of chondrogenic differentiation. The mRNA levels of eight genes were strongly correlated with MSC potency, as indicated by GAG production.

\section{Materials and Methods}

2.1. Cells. Human bone marrow MSCs were isolated from patients at the Hiroshima University Hospital and were cultured with the approval of the Hiroshima University Ethics Committee, as described previously [10, 16]. Ilium MSCs were isolated from 10 patients aged 25, 39, 53, 55, $59,61,63,64,65$, and 81 years, and jaw MSCs were collected from 5 patients aged 20, 28, 36, 36, and 63 years $[10,16]$. The donor ID numbers and ages are listed in Table S1 in Supplementary Materials.

2.2. Chondrogenic Differentiation of MSCs. MSCs from fourth-passage cultures were seeded at $2.5 \times 10^{5}$ cells in $15 \mathrm{~mL}$ centrifuge tubes for pellet culture in a chondrogenic differentiation medium and were maintained for 28 days as described previously $[10,17]$. The GAG contents were then measured using a sulfated GAG assay kit (Biocolor), according to the manufacturer's instructions. Data were normalized by the amounts of genomic DNA determined using PicoGreen fluorescence assays (Invitrogen).

2.3. Quantitative RT-PCR. Total RNA was isolated from confluent third-passage cultures using the RNeasy Mini Kit (Qiagen) as described previously [16]. First-strand cDNA was synthesized using ReverTra Ace- $\alpha$ (Toyobo), and realtime quantitative PCR was performed using the ABI Prism 7900 Sequence Detection System (Applied Biosystems) with a TaqMan low-density array (Applied Biosystems), which contains TaqMan probe and primer sets (TaqMan Gene Expression Assays) for 95 genes. The 95 genes were selected because their expression levels in ilium or jaw MSCs were more than two-fold higher than those in fibroblasts on microarray analyses [16]. The probe set IDs of TaqMan probe and primer sets for ACLY, AURKB, CD74, CDKN2D, E2F1, EFEMP1, LIF, and TGM2 are provided in Table 1. The genes examined in this study are listed in Table S1 in Supplementary Materials with their mRNA expression levels relative to that of $\beta$-actin.

2.4. Statistical Analysis. Statistical analyses were performed using SPSS Statistics for Windows, version 24.0. (IBM Corp.). Comparisons were made using Student's $t$-tests when comparing two experimental groups; correlations between mRNA expression levels and GAG contents, donor ages, or the mRNA expression levels of other genes were identified using Pearson correlation coefficients. The coefficient between the mRNA levels of group 2 genes and donor ages 


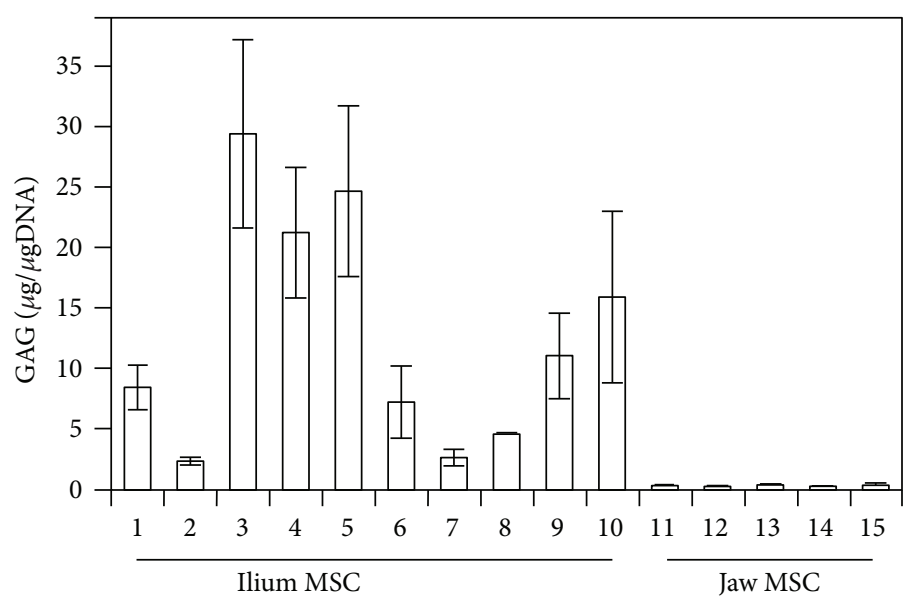

Figure 1: Chondrogenic differentiation potential of MSCs from ilium and jaw bone marrow. Chondrogenic differentiation levels were evaluated according to GAG contents at 28 days after induction. Data are presented as mean GAG contents normalized by genomic DNA amounts \pm standard errors of the mean from triplicate cultures; 1-10: ilium MSCs; 11-15: jaw MSCs.

TABLE 2: Correlation coefficients for expression levels of eight candidate predictive marker genes.

\begin{tabular}{|c|c|c|c|c|c|c|c|c|}
\hline Gene & $A U R K B$ & $E 2 F 1$ & $C D K N 2 D$ & LIF & $A C L Y$ & CD74 & EFEMP1 & TGM2 \\
\hline$A U R K B$ & 1.000 & & & & & & & \\
\hline E2F1 & $0.940^{* *}$ & 1.000 & & & & & & \\
\hline CDKN2D & $0.825^{* *}$ & $0.899^{* *}$ & 1.000 & & & & & \\
\hline LIF & $0.836^{* *}$ & $0.905^{* *}$ & $0.933^{* *}$ & 1.000 & & & & \\
\hline$A C L Y$ & $0.711^{* *}$ & $0.811^{* *}$ & $0.893^{* *}$ & $0.889^{* *}$ & 1.000 & & & \\
\hline CD74 & 0.244 & 0.406 & 0.314 & 0.401 & $0.571^{*}$ & 1.000 & & \\
\hline EFEMP1 & 0.396 & 0.483 & 0.366 & 0.369 & 0.502 & $0.827^{* *}$ & 1.000 & \\
\hline TGM2 & 0.343 & 0.431 & 0.315 & 0.419 & 0.494 & $0.751^{* *}$ & $0.785^{* *}$ & 1.000 \\
\hline
\end{tabular}

$r$ : Pearson correlation coefficient; ${ }^{*} P<0.05,{ }^{* *} P<0.01$. Strong Pearson correlation coefficient: $>0.75$.

were calculated by linear regression analysis. Differences and correlations were considered significant when $P<0.05$.

2.5. Cluster Analysis. Correlations between the mRNA expression levels of $A C L Y, A U R K B, C D 74, C D K N 2 D, E 2 F 1$, EFEMP1, LIF, and TGM2 were evaluated using hierarchical cluster analyses with the nearest-neighbor algorithm and are presented in a dendrogram that was generated using SPSS version 24.0.

2.6. Three-Dimensional (3D) Scatter Plot Analysis. 3D scatter plots were constructed using SPSS version 24.0. In this analysis, the relative mRNA expression levels of CDKN2D,CD74, and TGM2 in MSCs from 15 donors were recalculated relative to maximum values of 100 for each gene, and the distances between each point and the origin were then calculated.

\section{Results}

3.1. Chondrogenic Differentiation Potential of Ilium and Jaw MSCs. The potential and direction of differentiation vary depending on the source of the MSCs. In our previous study, we found that jaw MSCs have modest chondrogenic differentiation capacity compared with MSCs from the ilium $[5,16]$. Accordingly, the GAG contents of the pellet cultures of ilium MSCs (lanes 1-10) were much higher (2.3 to $29.4 \mu \mathrm{g})$ than those of the pellet cultures of jaw MSCs (lanes 11-15; $<0.3 \mu \mathrm{g})$ at 28 days after induction of chondrogenesis (Figure 1). However, the GAG contents varied widely even among ilium MSC cultures.

3.2. Correlations between Gene Expression Levels in Undifferentiated MSCs and GAG Contents after Induction of Chondrogenesis. The differences in chondrogenic differentiation potential between the ilium and jaw MSCs may help identify marker genes that can predict the MSC potential before induction. Thus, we assessed the correlations between gene expression levels in 15 MSC cultures (10 from ilium tissues and 5 from jaw tissues) and their differentiation levels after chondrogenic induction. We quantified the mRNA levels of 95 marker candidate genes using TaqMan low-density arrays [16] and analyzed the correlation between these levels and GAG contents. The mRNA expression patterns of eight of the 95 genes were significantly correlated with the GAG contents after induction (Table S1 in Supplementary Materials and Table 1); therefore, we selected these as candidate predictors of differentiation potential.

3.3. Correlation Analysis of Expression Levels of Eight Genes with Predictive Potential. To further examine the relationships between the identified eight genes, we correlated expression profiles in 15 MSC cultures (Table 2). These 


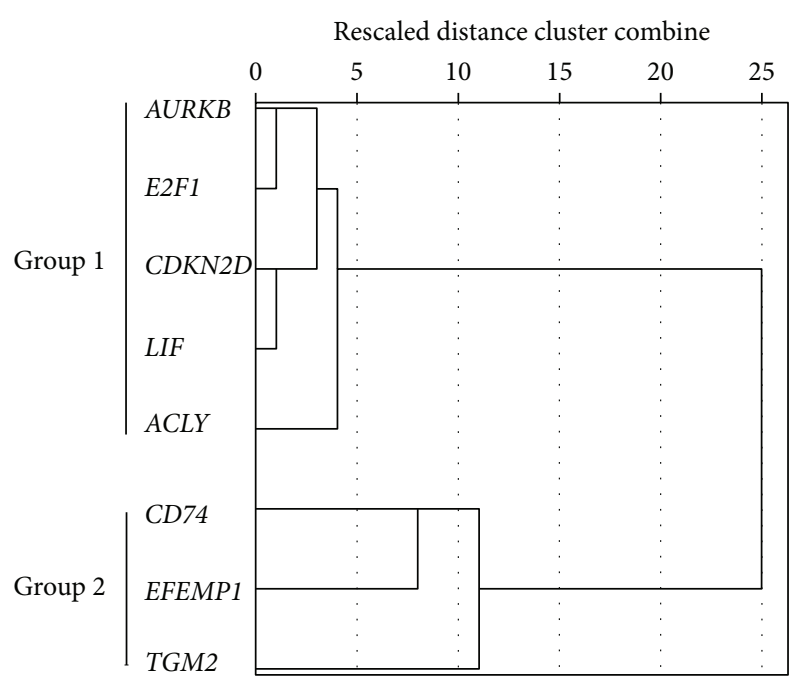

Figure 2: Dendrogram of the expression profiles of eight candidate prediction marker genes. Hierarchical cluster analysis of gene expression levels was performed using the nearestneighbor algorithm of SPSS. The horizontal dendrogram shows rescaled cumulative distances of clusters. The eight identified genes were classified into groups 1 and 2 according to the gene expression profiles.

analyses showed significant correlations in several combinations, presumably reflecting common regulatory mechanisms. To confirm these assumptions, we performed hierarchical cluster analyses using the nearest-neighbor algorithm and generated a dendrogram (Figure 2) that represents the overall relationships between the eight genes. Based on these correlation patterns, AURKB, E2F1, CDKN2D, LIF, and $A C L Y$ were allocated to group 1 and CD74, EFEMP1, and TGM2 were allocated to group 2, suggesting the presence of two predominant signaling pathways that determine chondrogenic differentiation potential.

3.4. Comparisons of Gene Expression Levels between Ilium and Jaw MSCs. Because the GAG contents were consistently lower in jaw MSCs than in those from the ilium, we compared the expression levels of the eight identified genes in MSCs from both tissues. These analyses revealed significantly higher mRNA expression levels of all eight genes in ilium MSCs than in jaw MSCs (Figure 3). Moreover, the expression levels of CDKN2D, CD74, and TGM2 were 22-, 18-, and 10 -fold higher, respectively, in ilium MSCs than in jaw MSCs. Thus, we selected these three genes as potential markers for further combined analyses.

3.5. Combined 3D Analysis of CDKN2D, CD74, and TGM2 Expression Levels. Although the CDKN2D, CD74, and TGM2 expression levels were much higher in ilium MSCs than in jaw MSCs, these considerably varied among the cultures of ilium MSCs. Thus, to predict MSC potential, we performed combined $3 \mathrm{D}$ analyses of expression levels and generated a $3 \mathrm{D}$ scatter plot for the three genes (Figure 4(a)). Distances from the origin for 9 out of 10 ilium MSCs (66.6 to 156.6) were greater than those for all jaw MSCs (2.8 to 13.4), whereas that for ilium MSC-4 was only 19.9 (Figure 4(b)). In addition, variations in combined expression data (Figure 5(a)) for all three genes were much smaller than for individual genes (Figure 5(b)). Hence, these combined $3 \mathrm{D}$ analyses may provide a more useful standard for predicting chondrogenic differentiation.

3.6. Negative Correlations between Gene Expression Levels and Donor Ages. We previously identified age-dependent decreases in chondrogenic differentiation potential of MSCs [10]. Thus, to evaluate the effects of age on the expression levels of the present eight genes with predictive potential, we correlated donor ages and mRNA expression levels in MSCs. The expression levels of the group 2 genes CD74, EFEMP1, and TGM2 were negatively correlated with donor ages (Table 3 and Figure 6), whereas no significant correlations were found for group 1 genes, suggesting that group 2 genes play predominant roles in age-dependent MSC chondrogenic differentiation potential.

\section{Discussion}

The predictions of MSC differentiation direction and potential are critical for clinical applications because the ensuing multipotency varies among donors. Although the factors that dictate differentiation potential into specific cell types remain poorly understood, we found that after chondrogenic induction, GAG contents were consistently low in pellet cultures of all MSCs from jaw tissues compared with those from ilium MSCs. Further, we attempted to identify the genes expressed at high levels in MSCs fated to produce large amounts of GAG but not in those fated to produce only small amounts of GAG after chondrogenic induction.

To identify markers of chondrocyte differentiation potential in MSCs, we correlated the gene expression in undifferentiated MSC monolayer cultures with GAG production in MSC pellet cultures after differentiation into chondrocytes. In these expression analyses, eight genes were significantly correlated with GAG contents, and these were classified into two groups according to their transcription profiles. These cluster analyses suggest common transcriptional regulatory mechanisms. Accordingly, the group 1 genes AURKB [18], E2F1 [19-21], CDKN2D [22], LIF $[23,24]$, and $A C L Y$ [25] participate in cell cycle regulation, whereas the group 2 genes EFEMP1 [26, 27], CD74 $[24,28-30]$, and TGM2 $[31,32]$ are involved in the regulation of chondrogenesis.

EFEMP1 (also known as fibulin-3), an ECM protein, is specifically expressed in cartilage and acts as a negative regulator of chondrogenesis [26]. Accordingly, overexpression of EFEMP1 reportedly suppressed the expression of the chondrogenic proteins SOX9, type II collagen, and aggrecan, whereas knockdown of EFEMP1 facilitated chondrogenesis [27]. CD74 is a receptor for macrophage migration inhibitory factor [33], which upregulates SOX9 expression [34]. TGM2 catalyzes the crosslinking of proteins such as fibronectin $[31,32]$ and thereby plays important roles in chondrogenesis [35]. 

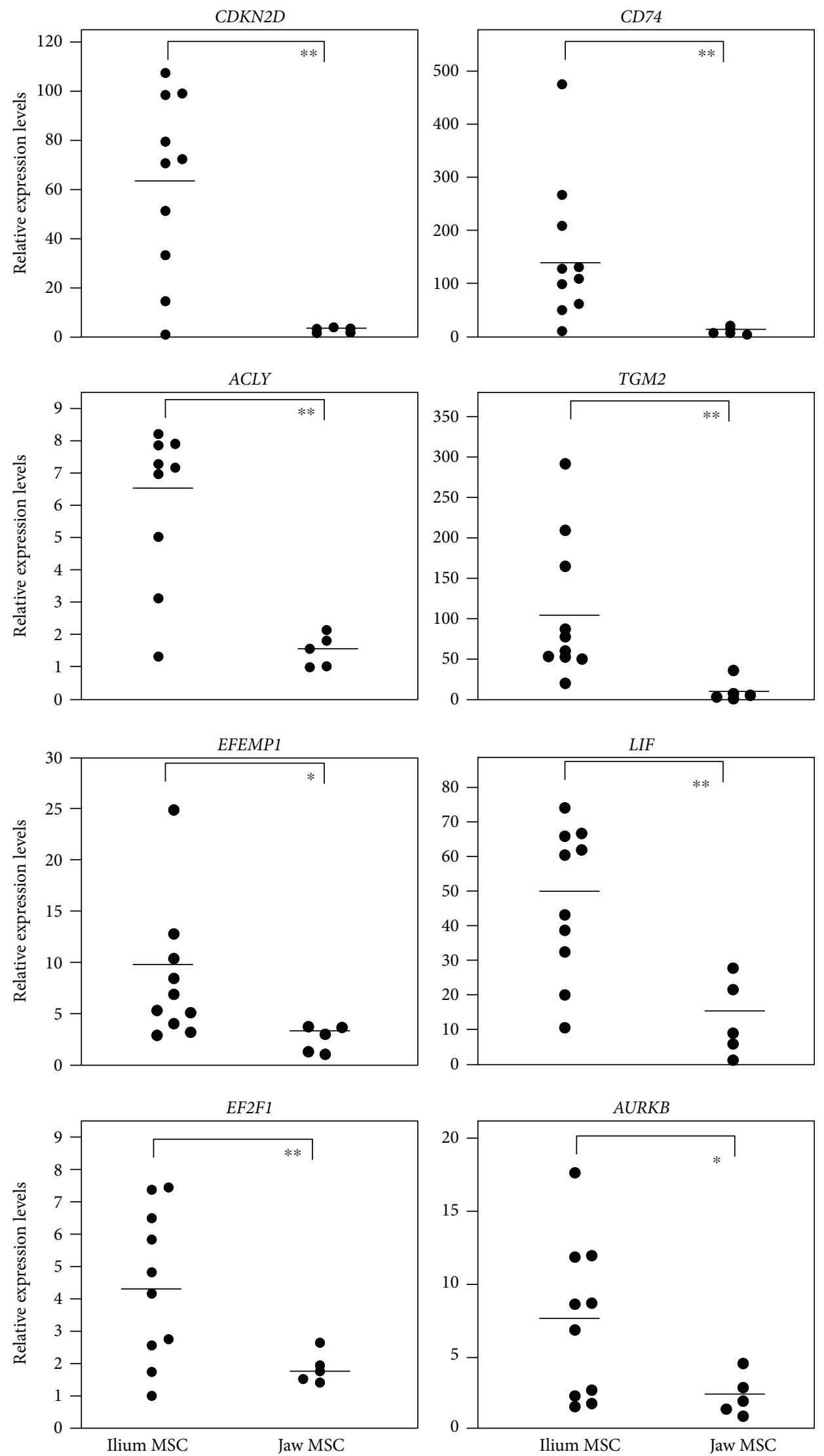

FIgURE 3: Comparisons of the expression levels of the eight candidate predictors of chondrogenesis between ilium MSCs $(n=10)$ and jaw MSCs $(n=5)$. Bars indicate mean values for each group; ${ }^{*} P<0.05,{ }^{* *} P<0.01$; Student's $t$-test. 


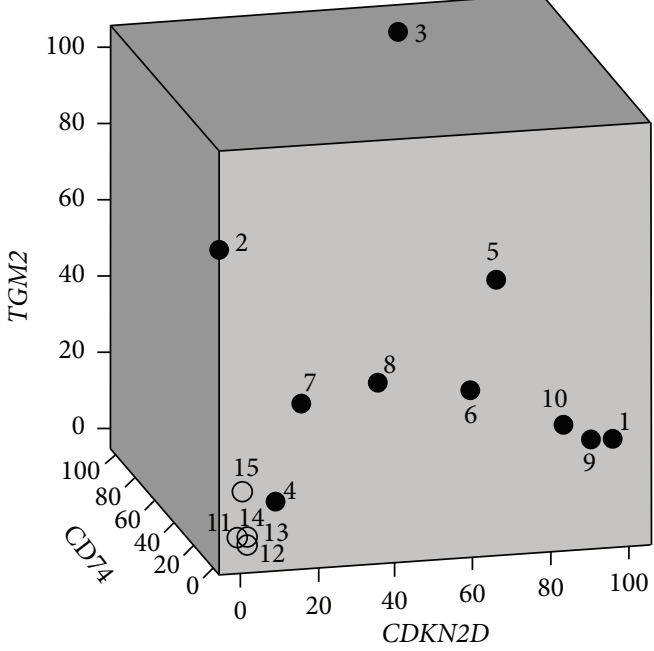

(a)

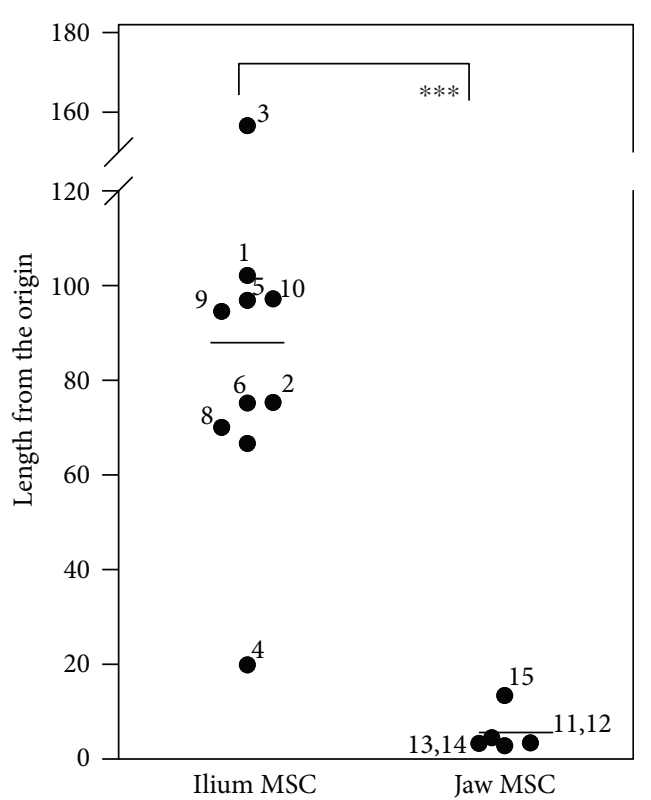

(b)

Figure 4: Combined 3D analysis of mRNA expression levels. (a) The scatter plot of CDKN2D ( $x$-axis), CD74 ( $y$-axis), and TGM2 ( $z$-axis) mRNA expression levels was constructed using SPSS. The relative mRNA expression levels in ilium (nos. 1-10) and jaw MSCs (nos. 11-15) are presented relative to maximum values of 100. (b) Mean distances between each point and the origin (bars) are shown for ilium and jaw MSCs. The donor ID numbers in the graphs correspond to those shown in Table S1 in Supplementary Materials; ${ }^{* * *} P<0.001$; Student's $t$-test.

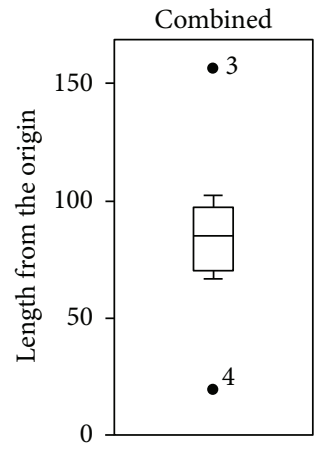

(a)
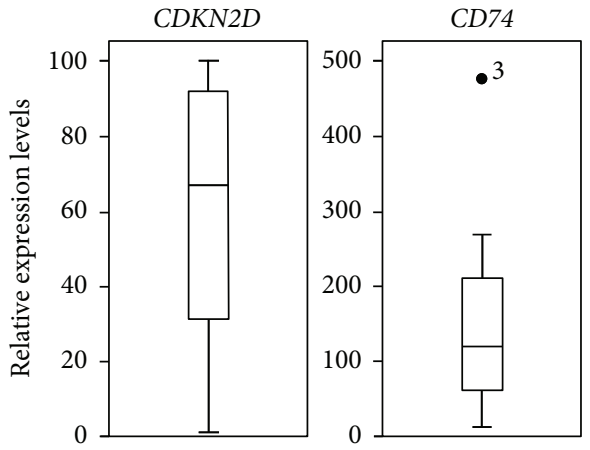

(b)

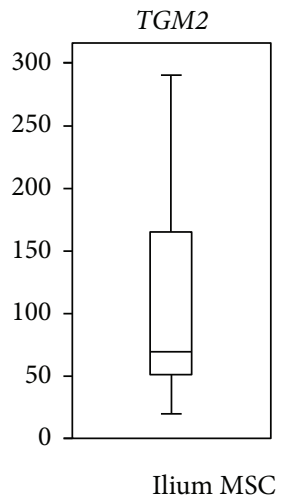

Figure 5: Comparisons of gene expression levels in ilium MSCs $(n=10)$. (a) Box plots show distances from the origin in combined 3D analysis of CDKN2D, CD74, and TGM2, as shown in Figure 4(a). (b) Box plots of CDKN2D, CD74, and TGM2 expression levels are presented with medians (bisecting line), interquartile ranges (box), 1.5-times interquartile ranges (whiskers), and outliers (dots). Outliers are numbered according to the donor IDs.

Among the eight genes identified herein, the mean CDKN2D, CD74, and TGM2 mRNA expression levels were more than 10-fold higher in ilium MSCs than in jaw MSCs. Although these varied widely between ilium MSC cultures, combined 3D analyses of these three genes showed promising results with very low variations and may represent a novel strategy for determining MSC differentiation potential.

MSCs are multipotent, and their fates are likely unpredictable, even if their potential for differentiation can be estimated. The present 3D combined analyses may offer promising assessments of differentiation potential, although the combined expression level in the MSC- 4 culture was relatively low, despite the high GAG contents. To compensate for this discrepancy, we performed additional twodimensional analysis of CDKN2D and CD74 (Figure S1 in Supplementary Materials), which showed much greater combined expression levels in ilium MSC-4 (no. 4) cultures than in jaw MSCs (nos. 11-15), similar combined expression to that in ilium MSC-2 (no. 2) cultures, but much lower combined expression than in the other ilium MSC cultures 
TABLE 3: Correlations between expression levels of candidate predictive marker genes and donor ages.

\begin{tabular}{lcc}
\hline Group & Gene & $r$ \\
\hline 1 & AURKB & 0.268 \\
1 & E2F1 & 0.083 \\
1 & CDKN2D & 0.363 \\
1 & LIF & 0.218 \\
1 & ACLY & 0.116 \\
2 & $C D 74$ & $-0.754^{*}$ \\
2 & EFEP1 & $-0.702^{*}$ \\
2 & TGM2 & $-0.852^{* *}$ \\
\hline
\end{tabular}

$r$ : Pearson correlation coefficients; ${ }^{*} P<0.05,{ }^{* *} P<0.01$.

(nos. 1, 3, and 5-10). Although this approach is sensitive to the expression of multiple genes, further studies of multiple MSC cultures from various tissues at various passages are required and could include fat- and synovium-derived MSCs.

In the present study, we used only 10 ilium and 5 jaw MSC lines owing to limited availability of MSC lines. Variations of the expression levels of the eight genes in ilium MSCs were much larger than those in jaw MSCs. Fortunately, we could examine 10 ilium MSC lines compared with 5 jaw MSC lines. In future research, the number of MSC lines should be increased to confirm our present results.

Although donor age strongly influences the potential for chondrogenic differentiation of MSCs, we previously showed that it is not a predictor of osteogenic or adipogenic differentiation [10]. In this study, the expression levels of the group 2 genes EFEMP1, CD74, and TGM2 were negatively correlated with the donor ages, potentially indicating their characterized roles in chondrogenesis. Collectively, these findings suggest that the group 2 genes are responsible for age-dependent chondrogenic differentiation. In agreement, EFEMP1 expression in articular cartilage was previously shown to decrease in an age-dependent manner [27].

Recently, Bertolo et al. [36] demonstrated marked decreases in the chondrogenic differentiation potential of MSCs after passage 7 . In their study concerning 84 senescence-related genes, the expression levels of 14 genes, including E2F1 and CDKN2D, were more than two-fold lower at passage 9 than at passage 3. As shown herein, these data suggest that high-level expression of cell cycle regulators such as E2F1 and CDKN2D is a requirement for high chondrogenic differentiation potential. In a previous study, we also evaluated the expression levels of 20 MSC marker genes, including LIF, CD74, and TGM2, from passages 1 to 10 [16]. Notably, LIF maintained similar expression levels from passages 1 to 10 , but the expression level of CD74 was upregulated at passage 10 . Further, the expression level of TGM2 at passage 1 is much higher than those from passages 2 to 10 . Thus, the expression levels of the marker genes found in this study showed various patterns. The relationship between expression levels of the eight genes and decreases in the chondrogenic differentiation potential after several passages should be analyzed in future research.
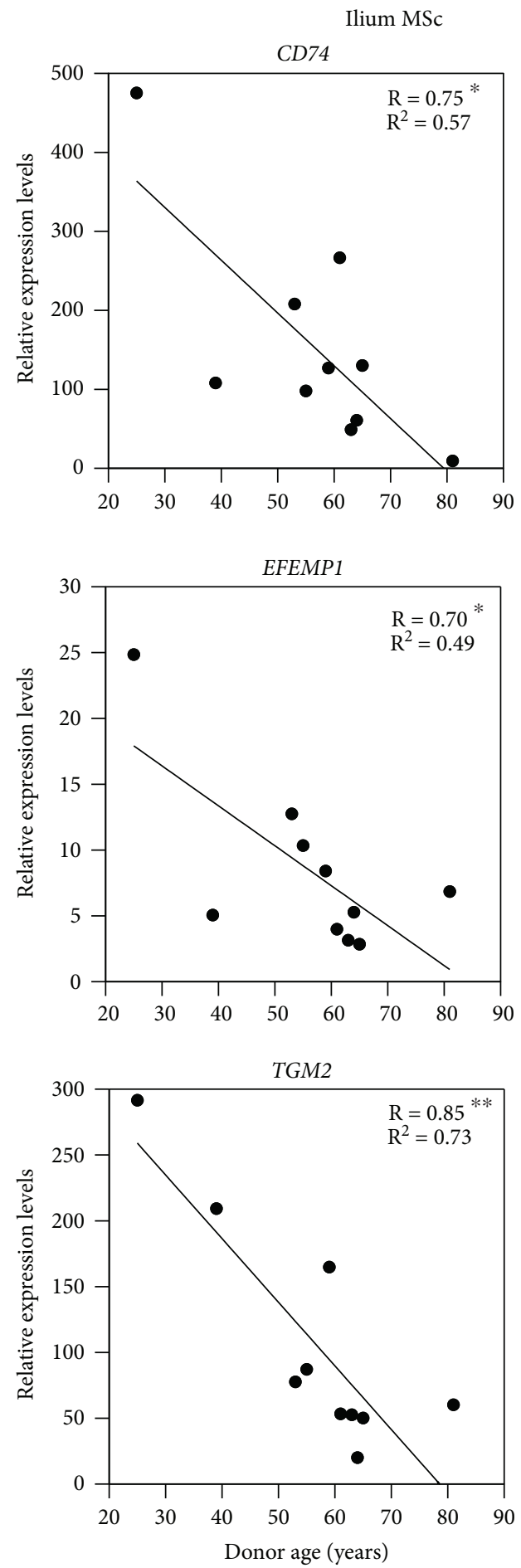

FIGURE 6: Regression analyses of the expression levels of the group 2 genes CD74, EFEMP1, and TGM2 in ilium MSCs and the donor ages. $n=10 ;{ }^{*} P<0.05,{ }^{* *} P<0.01$. R: multiple correlation coefficient; $\mathrm{R}^{2}$ : coefficient of determination.

We previously identified several genes with potential as MSC markers [16], and among these, TGM2 and LIF expressions were downregulated after the induction of chondrogenic differentiation [37]. Thus, markers of differentiation potential likely differ from markers of differentiation such 
as type II collagen and aggrecan, which are upregulated only after chondrogenic differentiation [38]. Therefore, while the markers identified herein indicate MSC potential, their expression levels are unlikely to distinguish between the degrees of differentiation.

Several cell surface markers, including CD105, CD146, CD271, and receptor tyrosine kinase-like orphan receptor 2 (ROR2), have been considered as markers of MSCs with high potential for chondrocyte differentiation [11-14]. However, to evaluate the expression levels of cell surface markers, we need experiments using fluorescenceactivated cell sorter (FACS) analysis, which uses suitable antigens for detecting cell surface molecules and single cells detached from culture dishes. The nature of MSCs detached from the dishes may differ from that of cells attached to the dishes. In addition, evaluation using FACS analysis is not quantitative, although FACS can be used to select a subpopulation that expresses high levels of a cell surface marker. Among these cell surface markers, the CD146 (MCAM) gene was included in the 95 genes evaluated in this study (Table S1 in Supplementary Materials). CD146 showed a similar expression pattern as the eight genes identified in this study. Its expression level in ilium MSCs was significantly higher than that in jaw MSCs (data not shown), although the correlation of CD146 expression with GAG production was not significant, suggesting that the eight genes are more reliable markers than cell surface markers such as CD146. Thus, measuring the prediction marker expression levels in cells attached to the dishes could be desirable for estimating the differentiation potential of MSCs.

\section{Conclusions}

The present 3D expression analyses of predictive marker genes offer a novel strategy for assessing MSC differentiation potential and could form the basis for predicting clinical outcomes of MSC therapy.

\section{Data Availability}

The data used to support the findings of this study are available from the corresponding author upon request.

\section{Conflicts of Interest}

The authors declare that there is no conflict of interest regarding the publication of this paper.

\section{Funding}

This work was supported by JSPS KAKENHI (JP17K11541).

\section{Supplementary Materials}

Figure S1: combined two-dimensional analysis of CD74 and CDKN2D mRNA expression levels. Table S1: correlation coefficients for 95 gene expression levels in undifferentiated MSC and GAG contents after chondrogenic induction, along with donor IDs and ages. (Supplementary Materials.)

\section{References}

[1] M. F. Pittenger, A. M. Mackay, S. C. Beck et al., "Multilineage potential of adult human mesenchymal stem cells," Science, vol. 284, no. 5411, pp. 143-147, 1999.

[2] R. M. Samsonraj, M. Raghunath, V. Nurcombe, J. H. Hui, A. J. van Wijnen, and S. M. Cool, "Concise review: multifaceted characterization of human mesenchymal stem cells for use in regenerative medicine," Stem Cells Translational Medicine, vol. 6, no. 12, pp. 2173-2185, 2017.

[3] A. Uccelli, L. Moretta, and V. Pistoia, "Mesenchymal stem cells in health and disease," Nature Reviews Immunology, vol. 8, no. 9, pp. 726-736, 2008.

[4] R. J. Deans and A. B. Moseley, "Mesenchymal stem cells: biology and potential clinical uses," Experimental Hematology, vol. 28 , no. 8, pp. 875-884, 2000.

[5] T. Matsubara, K. Suardita, M. Ishii et al., "Alveolar bone marrow as a cell source for regenerative medicine: differences between alveolar and iliac bone marrow stromal cells," Journal of Bone and Mineral Research, vol. 20, no. 3, pp. 399-409, 2005.

[6] L. Xu, Y. Liu, Y. Sun et al., "Tissue source determines the differentiation potentials of mesenchymal stem cells: a comparative study of human mesenchymal stem cells from bone marrow and adipose tissue," Stem Cell Research \& Therapy, vol. 8, no. 1, p. 275, 2017.

[7] R. N. Bearden, S. S. Huggins, K. J. Cummings, R. Smith, C. A. Gregory, and W. B. Saunders, "In-vitro characterization of canine multipotent stromal cells isolated from synovium, bone marrow, and adipose tissue: a donor-matched comparative study," Stem Cell Research \& Therapy, vol. 8, no. 1, p. 218, 2017.

[8] D. L. Deskins, D. Bastakoty, S. Saraswati, A. Shinar, G. E. Holt, and P. P. Young, "Human mesenchymal stromal cells: identifying assays to predict potency for therapeutic selection," Stem Cells Translational Medicine, vol. 2, no. 2, pp. 151-158, 2013.

[9] A. Mentink, M. Hulsman, N. Groen et al., "Predicting the therapeutic efficacy of MSC in bone tissue engineering using the molecular marker CADM1," Biomaterials, vol. 34, no. 19, pp. 4592-4601, 2013.

[10] M. Kanawa, A. Igarashi, V. S. Ronald et al., "Age-dependent decrease in the chondrogenic potential of human bone marrow mesenchymal stromal cells expanded with fibroblast growth factor-2," Cytotherapy, vol. 15, no. 9, pp. 1062-1072, 2013.

[11] W. Fan, J. Li, Y. Wang et al., "CD105 promotes chondrogenesis of synovium-derived mesenchymal stem cells through Smad2 signaling," Biochemical and Biophysical Research Communications, vol. 474, no. 2, pp. 338-344, 2016.

[12] Y. X. Wu, X. Z. Jing, Y. Sun et al., "CD146 ${ }^{+}$skeletal stem cells from growth plate exhibit specific chondrogenic differentiation capacity in vitro," Molecular Medicine Reports, vol. 16, no. 6, pp. 8019-8028, 2017.

[13] Y. Mifune, T. Matsumoto, S. Murasawa et al., "Therapeutic superiority for cartilage repair by CD271-positive marrow stromal cell transplantation," Cell Transplantation, vol. 22, no. 7, pp. 1201-1211, 2013.

[14] S. C. Dickinson, C. A. Sutton, K. Brady et al., "The Wnt5a receptor, receptor tyrosine kinase-like orphan receptor 2 , is a predictive cell surface marker of human mesenchymal stem cells with an enhanced capacity for chondrogenic differentiation," Stem Cells, vol. 35, no. 11, pp. 2280-2291, 2017. 
[15] C. C. Wu, F. L. Liu, H. K. Sytwu, C. Y. Tsai, and D. M. Chang, "CD $146^{+}$mesenchymal stem cells display greater therapeutic potential than $\mathrm{CD}_{146^{-}}$cells for treating collagen-induced arthritis in mice," Stem Cell Research \& Therapy, vol. 7, no. 1, p. $23,2016$.

[16] A. Igarashi, K. Segoshi, Y. Sakai et al., "Selection of common markers for bone marrow stromal cells from various bones using real-time RT-PCR: effects of passage number and donor age," Tissue Engineering, vol. 13, no. 10, pp. 2405-2417, 2007.

[17] R. W. Farndale, C. A. Sayers, and A. J. Barrett, "A direct spectrophotometric microassay for sulfated glycosaminoglycans in cartilage cultures," Connective Tissue Research, vol. 9, no. 4, pp. 247-248, 1982.

[18] A. Szedlak, S. Sims, N. Smith, G. Paternostro, and C. Piermarocchi, "Cell cycle time series gene expression data encoded as cyclic attractors in Hopfield systems," PLoS Computational Biology, vol. 13, no. 11, article e1005849, 2017.

[19] B. Scheijen, M. Bronk, T. van der Meer, and R. Bernards, "Constitutive E2F1 overexpression delays endochondral bone formation by inhibiting chondrocyte differentiation," Molecular and Cellular Biology, vol. 23, no. 10, pp. 3656-3668, 2003.

[20] M. C. Chen, Y. C. Tsai, J. H. Tseng et al., "Simvastatin inhibits cell proliferation and migration in human anaplastic thyroid cancer," International Journal of Molecular Sciences, vol. 18, no. 12, 2017.

[21] H. Müller and K. Helin, "The E2F transcription factors: key regulators of cell proliferation," Biochimica et Biophysica Acta (BBA) - Reviews on Cancer, vol. 1470, no. 1, pp. M1-M12, 2000.

[22] Y. Wang, W. Jin, X. Jia et al., "Transcriptional repression of CDKN2D by PML/RAR $\alpha$ contributes to the altered proliferation and differentiation block of acute promyelocytic leukemia cells," Cell Death \& Disease, vol. 5, no. 10, article e1431, 2014.

[23] S. A. Ali, G. Kaur, J. K. Kaushik, D. Malakar, A. K. Mohanty, and S. Kumar, "Examination of pathways involved in leukemia inhibitory factor (LIF)-induced cell growth arrest using labelfree proteomics approach," Journal of Proteomics, vol. 168, pp. 37-52, 2017.

[24] L. Wu, C. Bluguermann, L. Kyupelyan et al., "Human developmental chondrogenesis as a basis for engineering chondrocytes from pluripotent stem cells," Stem Cell Reports, vol. 1, no. 6, pp. 575-589, 2013.

[25] N. Zaidi, I. Royaux, J. V. Swinnen, and K. Smans, “ATP citrate lyase knockdown induces growth arrest and apoptosis through different cell- and environment-dependent mechanisms," Molecular Cancer Therapeutics, vol. 11, no. 9, pp. 1925-1935, 2012.

[26] T. Wakabayashi, A. Matsumine, S. Nakazora et al., "Fibulin-3 negatively regulates chondrocyte differentiation," Biochemical and Biophysical Research Communications, vol. 391, no. 1, pp. 1116-1121, 2010.

[27] A. Hasegawa, T. Yonezawa, N. Taniguchi et al., "Role of fibulin 3 in aging-related joint changes and osteoarthritis pathogenesis in human and mouse knee cartilage," Arthritis \& Rheumatology, vol. 69, no. 3, pp. 576-585, 2017.

[28] C. Xiong, B. Huang, Y. Cun, B. G. Aghdasi, and Y. Zhou, "Migration inhibitory factor enhances inflammation via CD74 in cartilage end plates with Modic type 1 changes on MRI," Clinical Orthopaedics and Related Research, vol. 472, no. 6, pp. 1943-1954, 2014.
[29] H. Sternberg, J. T. Murai, I. E. Erickson et al., "A human embryonic stem cell-derived clonal progenitor cell line with chondrogenic potential and markers of craniofacial mesenchyme," Regenerative Medicine, vol. 7, no. 4, pp. 481-501, 2012.

[30] M. Ishii, C. Koike, A. Igarashi et al., "Molecular markers distinguish bone marrow mesenchymal stem cells from fibroblasts," Biochemical and Biophysical Research Communications, vol. 332, no. 1, pp. 297-303, 2005.

[31] S. S. Akimov and A. M. Belkin, "Cell-surface transglutaminase promotes fibronectin assembly via interaction with the gelatinbinding domain of fibronectin: a role in TGF $\beta$-dependent matrix deposition," Journal of Cell Science, vol. 114, no. 16, pp. 2989-3000, 2001.

[32] A. M. Belkin, "Extracellular TG2: emerging functions and regulation," The FEBS Journal, vol. 278, no. 24, pp. 4704-4716, 2011.

[33] L. Leng, C. N. Metz, Y. Fang et al., "MIF signal transduction initiated by binding to CD74," Journal of Experimental Medicine, vol. 197, no. 11, pp. 1467-1476, 2003.

[34] Y. Yao, Q. Deng, W. Song et al., "MIF plays a key role in regulating tissue-specific chondro-osteogenic differentiation fate of human cartilage endplate stem cells under hypoxia," Stem Cell Reports, vol. 7, no. 2, pp. 249-262, 2016.

[35] P. Singh and J. E. Schwarzbauer, "Fibronectin and stem cell differentiation-lessons from chondrogenesis," Journal of Cell Science, vol. 125, no. 16, pp. 3703-3712, 2012.

[36] A. Bertolo, M. Mehr, T. Janner-Jametti et al., "An in vitro expansion score for tissue-engineering applications with human bone marrow-derived mesenchymal stem cells," Journal of Tissue Engineering and Regenerative Medicine, vol. 10, no. 2, pp. 149-161, 2016.

[37] H. Kubo, M. Shimizu, Y. Taya et al., "Identification of mesenchymal stem cell (MSC)-transcription factors by microarray and knockdown analyses, and signature molecule-marked MSC in bone marrow by immunohistochemistry," Genes to Cells, vol. 14, no. 3, pp. 407-424, 2009.

[38] K. Pelttari, E. Steck, and W. Richter, "The use of mesenchymal stem cells for chondrogenesis," Injury, vol. 39, no. 1, pp. 58-65, 2008 . 


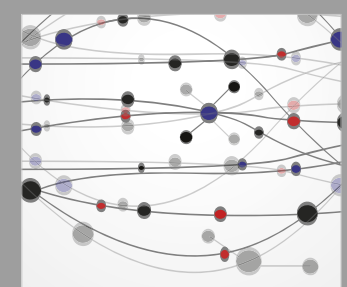

The Scientific World Journal
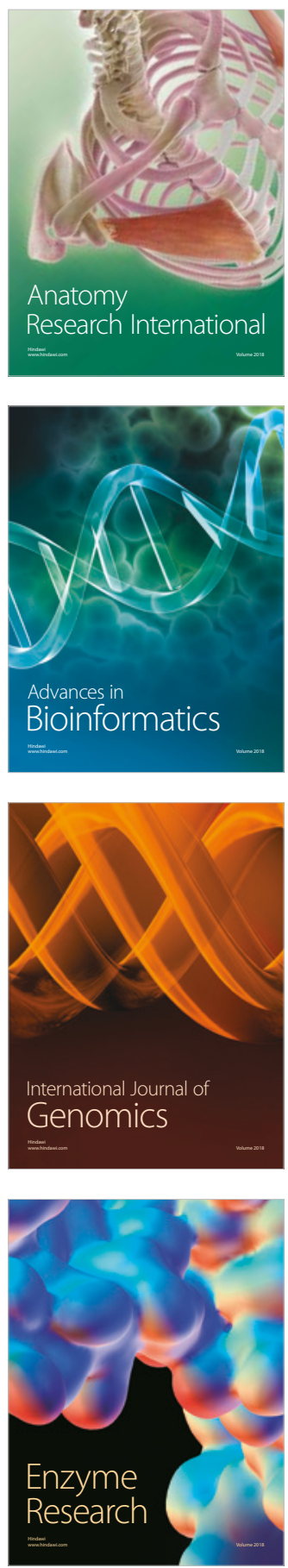
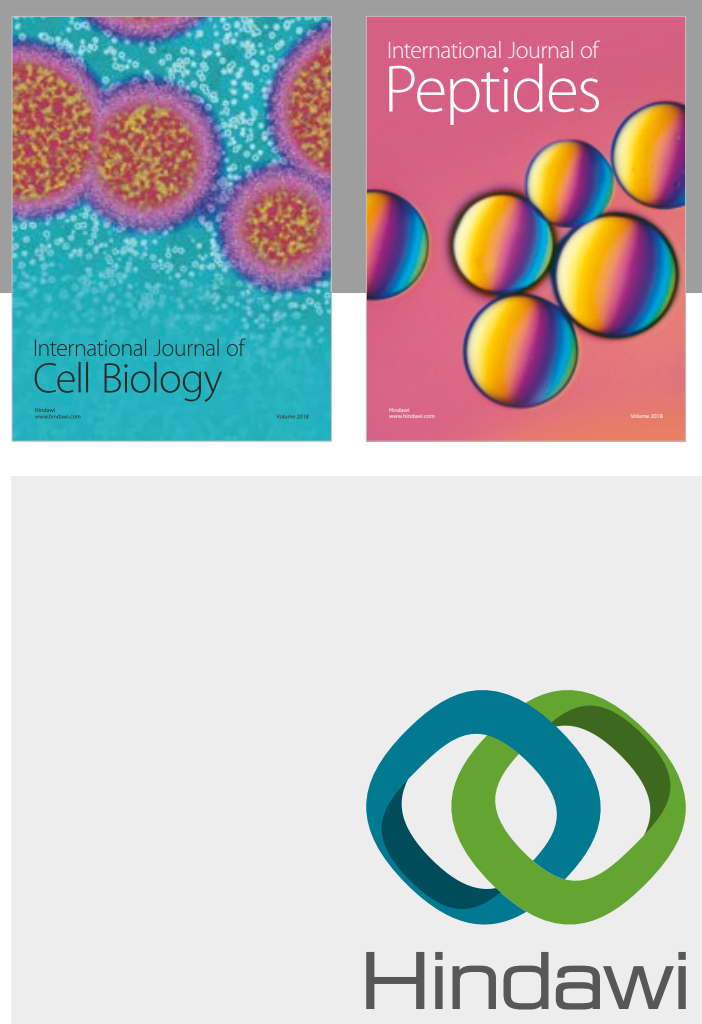

Submit your manuscripts at

www.hindawi.com
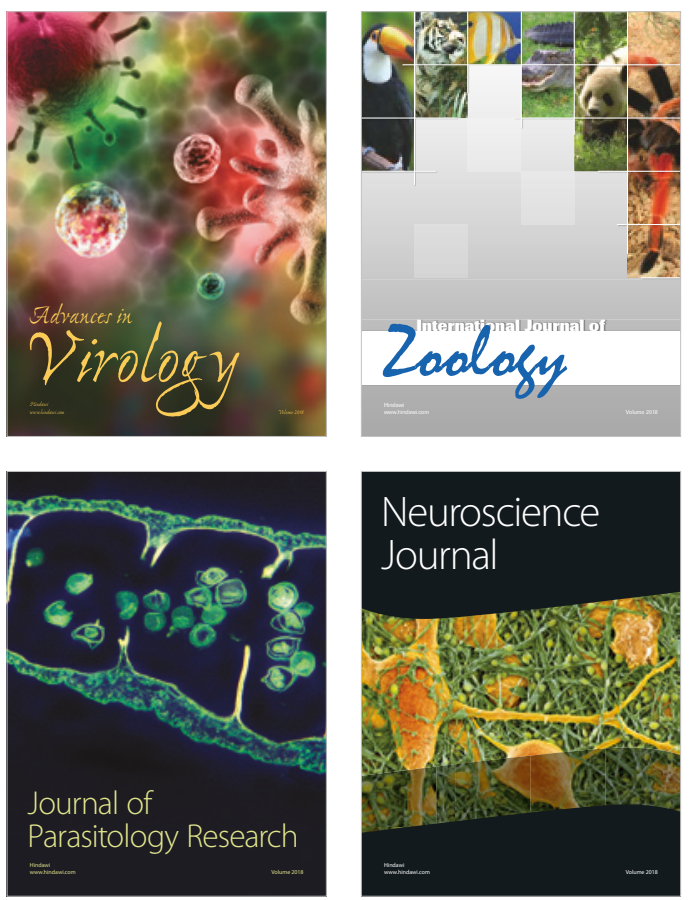
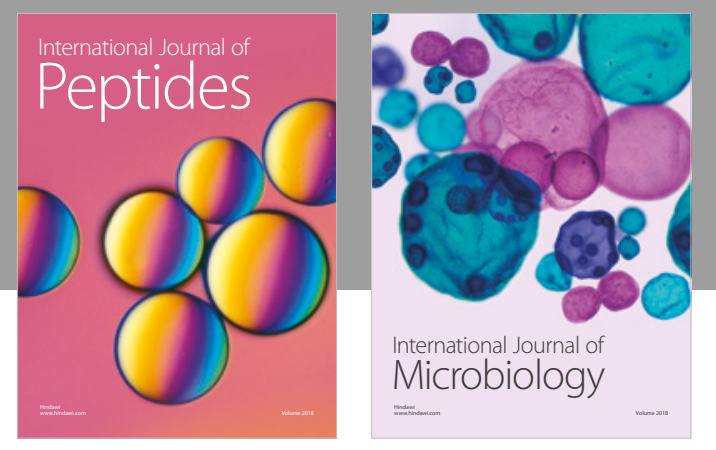

nternational Journal of Microbiology
Journal of
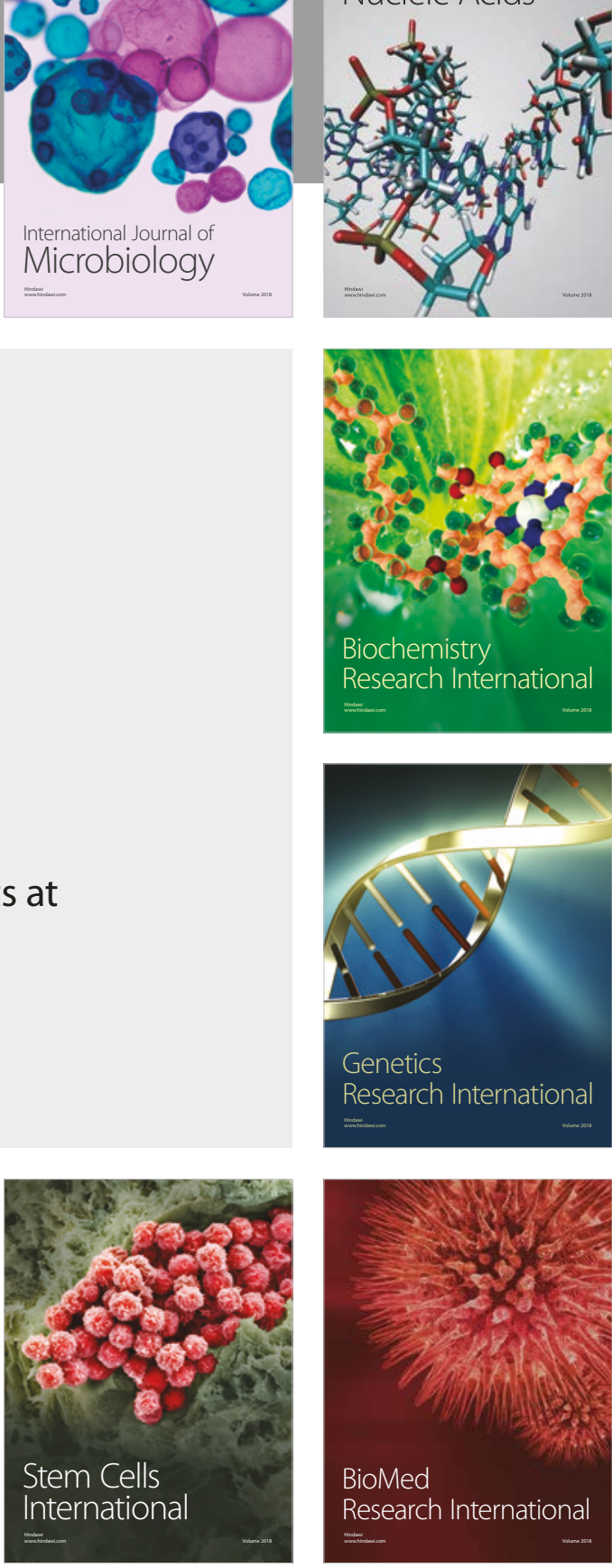
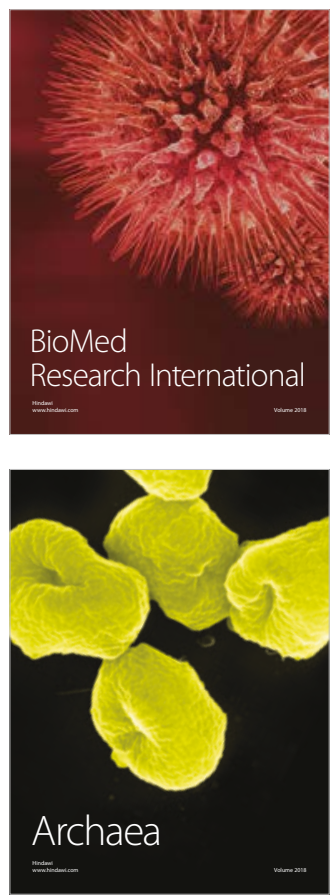